\title{
Corrosion Damage of Copper Tubes
}

\author{
Michael Panzenböck, *
}

* Department of Physical Metallurgy and Materials Testing, University of Leoben, Austria

As it is well known, copper is a very corrosion resistant material. Because of this special feature the material is used for buildings, facades and roofs, heatings as well as for warm- and cold-water installations. Sometimes failure occurs in such water installations. The damage which often penetrates through the full wall thickness of the tubes depends mainly on the interaction between material and environment. Here the chemical composition of the tubes, manufacturing processes and the workmanship play a dominant role. In many cases pits and cracks can be observed on the outer or inner surface. In this contribution two typical failure mechanisms will be discussed.

Corrosion resistance of metals can be either very good or quite poor depending on the environment. In general, "ignoble metals" such as iron heavily corrode in the moist air whereas "noble metals" exhibit no surface damage. But it is also known that many "ignoble metals" e.g. aluminium, magnesium, zinc etc. show a very high resistance against corrosion under normal conditions. The reason for this is a well bonded dense layer of corrosion products consisting of oxides and/or carbonates. Such layers can be also observed on copper. These two aspects - passive layer and "noble metal" (electromotive series) - are the reason why copper is used in many fields e.g. architecture, as a roofing material and in service lines. However the picture of copper as a high corrosion resistant material will be marred after occurrence of leaks in heating systems or cold and warm water supplies. It can be imagined that local destructions of water filled tubes lead to high repair costs.

First of all many cracks on the outer surface of copper tubes used for a cold water installation were found in the longitudinal direction (Fig. 1). Careful investigations show that the crack initiation starts at the outside of the tubes. This reveals Fig. 2 where the main crack together with many branched cracks is visible. Crack propagation occurs only along the grain boundaries. A direct view of the main crack (Fig. 3) shows separated grains, secondary cracks and corrosion products. All these facts indicate that the damage is caused by stress corrosion cracking (SCC). Chemical investigations suggest that sodium nitrite and calcium nitrite were responsible for the damage. Nevertheless tension stresses are also necessary for SCC. A simple method allows an estimation of the residual stresses. After cutting tubes in the longitudinal direction the cutting clearance will open in a case when the circumferential tension stresses are present. In other words, the circumference of the tubes will change. Taking all geometry factors into account, a stress level of about $130 \mathrm{MPa}$ was calculated.

Another predominant failure mechanism of copper tubes is pitting corrosion. After new installation of the water supply in an old building, water patches on the wall were observed within one year. The damaged parts (Fig. 4) of the water pipe were cut out and replaced with new tubes. Soft-soldering techniques were used for connecting the pipes and fittings. Subsequently the pipes were flushed with citric acid. Such a procedure has to be repeated two or three times a year. The house seems to be an everlasting construction site. Many local attacks are predominant in the vicinity of the pipe connection (Fig. 5, 6). Cuprite and malachite are detectable within these pits. In addition, residues of soldering flux were found at the inner surface of the tubes. Thin films of carbon layers and chloride containing soldering facilities were responsible for the disastrous failure of the pipes. 


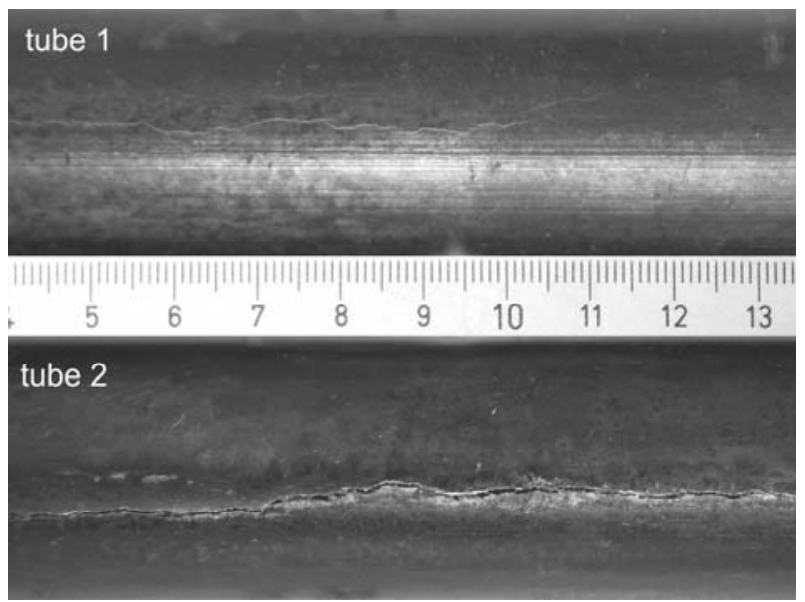

FIG. 1. Outer surface of two tubes with cracks in the longitudinal direction

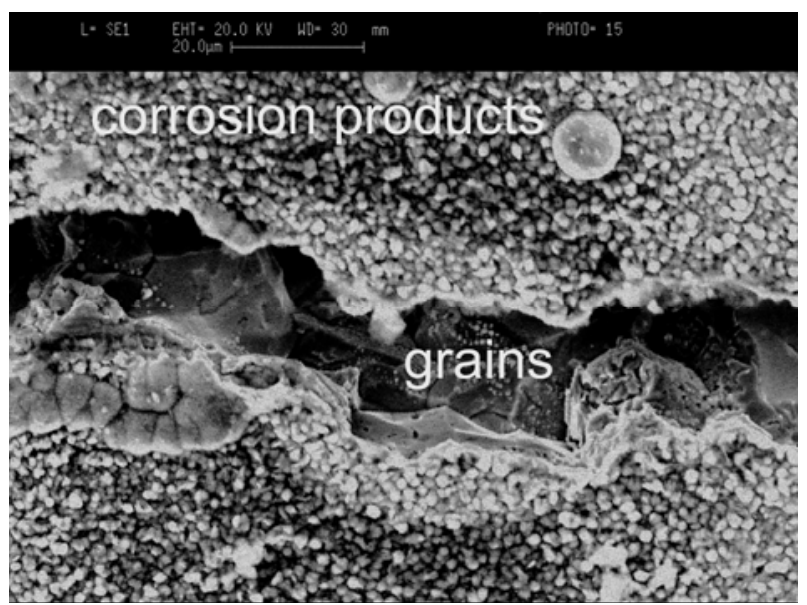

FIG. 3. Overview of the main crack at the outer surface of the tube

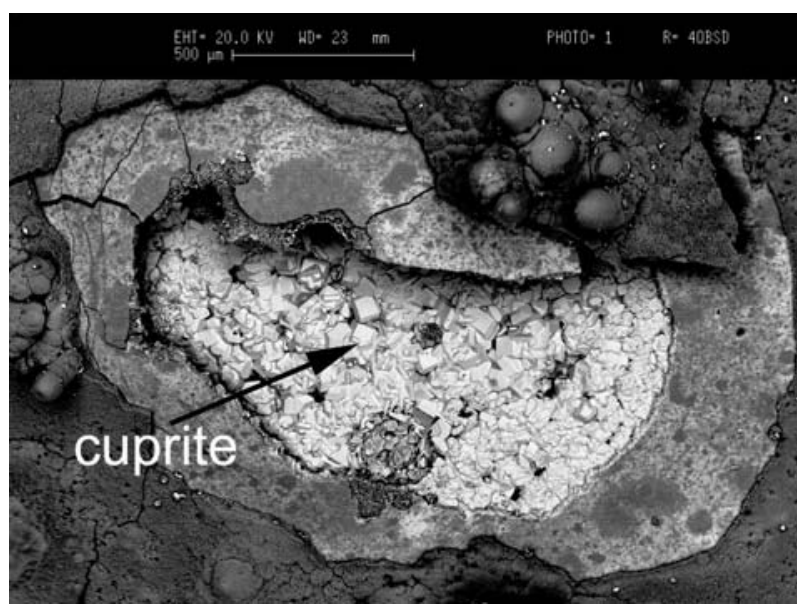

FIG. 5. Local attack at the inner surface of the copper tube

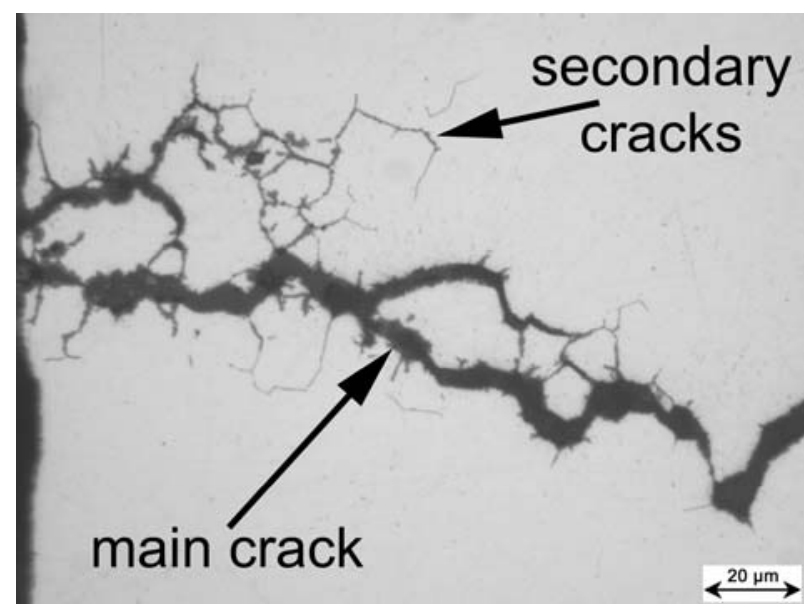

FIG. 2. Microstructure of the copper tube, the main crack starts from the outside

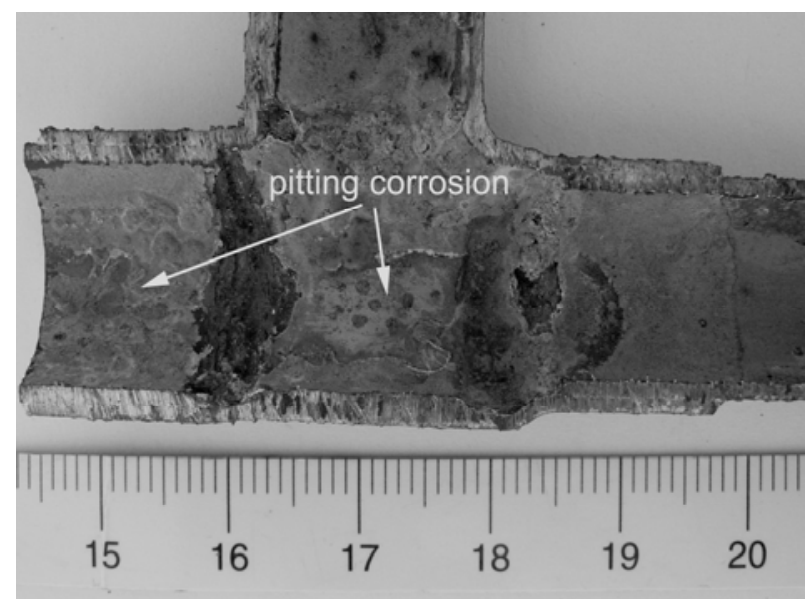

FIG. 4. Overview of the inside of the cooper tube, in the vicinity of the pipe connection

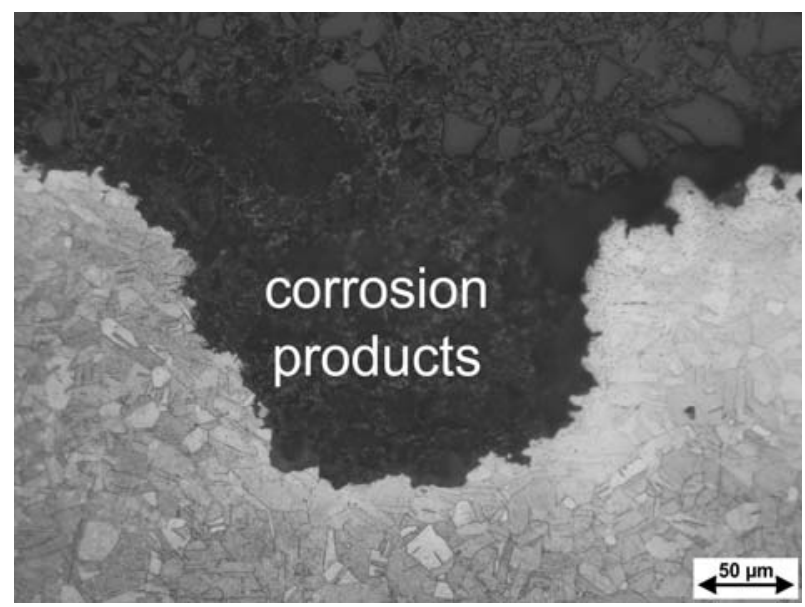

FIG. 6. Cross section of a local attack at the inner surface 